\title{
ESTUDO DA PRECIPITAÇÃO EM AÇOS DE ALTA RESISTÊNCIA E BAIXA LIGA POR TERMODINÂMICA COMPUTACIONAL
}

Meire Guimarães Lage Carlos Salaroli de Araújo ${ }^{2}$

\section{Resumo}

O presente trabalho teve como objetivo o estudo da precipitação em aços ARBL microligados ao $\mathrm{Ti}, \mathrm{Nb}$ e $\mathrm{V}$, visando dar subsídios ao desenvolvimento de projetos de liga e às etapas de processamento termomecânico. Para isso, foram realizados cálculos no programa computacional Thermo-Calc, versão 4, obtendo-se diagramas de equilíbrio, nos quais foram avaliadas a precipitação de compostos, as condições de solubilização de elementos na austenita e a influência dos elementos C, N, Nb e V sobre a precipitação. A metodologia de estudo forneceu informações qualitativas importantes sobre o fenômeno da precipitação, fundamentais para o estabelecimento de rotas de processamento termomecânico de aços ARBL.

Palavras-chave: Aço alta resistência; Aço baixa liga; Termodinâmica computacional; Precipitação.

\section{STUDY OF PRECIPITATION IN HIGH STRENGTH LOW ALLOY STEEL BY COMPUTATIONAL THERMODYNAMIC}

\begin{abstract}
The purpose of present work was to study the precipitation in $\mathrm{Ti}, \mathrm{Nb}$ and $\mathrm{V}$ microalloyed HSLA steels, aimed to provide subsides for alloy design and thermomechanical processing. Calculations were performed using the Thermo-Calc software, version 4. The precipitation of compounds, the conditions for dissolution of elements in the austenite and the influence of the elements $\mathrm{C}, \mathrm{N}, \mathrm{Nb}$ and $\mathrm{V}$ on precipitation were evaluated using equilibrium diagrams. The methodology of the study provided important qualitative information about the phenomenon of precipitation, which are fundamental to establish routes of HSLA thermomechanical process.
\end{abstract}

Key words: High strength steel; Low alloy steel; Computational thermodynamics; Precipitation.

\section{INTRODUÇÃO}

Aços de alta resistência e baixa liga (ARBL) são materiais estruturais que apresentam boa soldabilidade e elevadas resistência mecânica e tenacidade, sendo empregados nos setores naval, offshore e de tubos. Geralmente, são microligados com Ti, Nb e/ou V, que se combinam com $\mathrm{C}$ e $\mathrm{N}$ para formar carbonetos e nitretos ou um complexo destes precipitados, os carbonitretos. Os requisitos de propriedades mecânicas destes aços podem ser alcançados através do refino de grão e do endurecimento por precipitação, que são obtidos pela combinação da adição de elementos microligantes e do processamento termomecânico. ${ }^{(1)} \mathrm{O}$ conhecimento do fenômeno da precipitação é importante para a otimização do processamento termomecânico, visando alcançar os requisitos de propriedades mecânicas desejadas ao material. ${ }^{(2)}$ Por exemplo, a temperatura de reaquecimento é determinada para se garantir que todo ou grande parte do $\mathrm{Nb}$ adicionado permaneça em solução sólida na austenita e que o tamanho de grão desta fase seja impedido de crescer pela presença de precipitados finos de $\mathrm{TiN} .{ }^{(3)}$

Atualmente, a termodinâmica computacional está sendo utilizada no cálculo de diagramas de equilíbrio de fases de sistemas multicomponentes, auxiliando o desenvolvimento de projetos de ligas e a otimização das condições de processamento termomecânico. Um dos programas computacionais mais utilizados é o Thermo-Calc, que calcula a energia livre de Gibbs mínima das fases, usando funções ajustadas de vários dados termodinâmicos experimentais. ${ }^{(4)}$

\footnotetext{
'Membro da ABM, Engenheira Química, Mestre em Engenharia Metalúrgica e de Minas, Universidade Federal de Minas Gerais - UFMG, Centro de Tecnologia USIMINAS - Unidade Ipatinga. Av. Pedro Linhares Gomes, 543I, Bairro Usiminas, Cep 35I60-900, Ipatinga, MG, Brasil. E-mail:meire.lage@usiminas.com

${ }^{2}$ Membro da ABM, Engenheiro Metalurgista, Mestre em Engenharia Metalúrgica, Universidade Federal do Rio de Janeiro - UFRJ, Centro de Tecnologia USIMINAS - Unidade Ipatinga. Av. Pedro Linhares Gomes, 543I, Bairro Usiminas, Cep 35 I60-900, Ipatinga, MG, Brasil. E-mail: carlos.araujo@usiminas.com
} 
O presente trabalho teve como objetivo estudar a precipitação de elementos microligantes em aços ARBL, mediante emprego da termodinâmica computacional, visando dar subsídios ao desenvolvimento de projetos de liga e, também, às etapas de processamento termomecânico.

\section{MATERIAIS E MÉTODOS}

Foram avaliados distintos sistemas de ligas de aços ARBL, que diferem entre si pelos teores de $\mathrm{C}, \mathrm{N}, \mathrm{Nb}$ e pela adição de $\mathrm{V}$. Na Tabela I estão mostrados os sistemas e suas respectivas composições químicas.

Tabela I. Composição química dos sistemas de liga de aços ARBL.

\begin{tabular}{cccccccccc}
\hline \multirow{2}{*}{ Sistema } & \multicolumn{10}{c}{ Composição química (\%p) } \\
\cline { 2 - 10 } & C & Mn & Si & Al & Nb & Ti & V & N & Outros \\
\hline A & 0,05 & 1,60 & 0,30 & 0,030 & 0,045 & 0,013 & - & 0,0050 & $\mathrm{Mo}, \mathrm{Cu}, \mathrm{Cr}, \mathrm{Ni}$ \\
B & 0,10 & 1,60 & 0,30 & 0,030 & 0,045 & 0,013 & - & 0,0050 & $\mathrm{Mo}, \mathrm{Cu}, \mathrm{Cr}, \mathrm{Ni}$ \\
C & 0,05 & 1,60 & 0,30 & 0,030 & 0,015 & 0,013 & - & 0,0050 & $\mathrm{Mo}, \mathrm{Cu}, \mathrm{Cr}, \mathrm{Ni}$ \\
D & 0,05 & 1,60 & 0,30 & 0,030 & 0,045 & 0,013 & 0,050 & 0,0050 & $\mathrm{Mo}, \mathrm{Cu}, \mathrm{Cr}, \mathrm{Ni}$ \\
E & 0,10 & 1,60 & 0,30 & 0,030 & 0,045 & 0,013 & 0,050 & 0,0050 & $\mathrm{Mo}, \mathrm{Cu}, \mathrm{Cr}, \mathrm{Ni}$ \\
F & 0,05 & 1,60 & 0,30 & 0,030 & 0,045 & 0,013 & 0,050 & 0,0080 & $\mathrm{Mo}, \mathrm{Cu}, \mathrm{Cr}, \mathrm{Ni}$ \\
\hline
\end{tabular}

Os diagramas de equilíbrio de cada sistema de liga foram obtidos mediante cálculos no Thermo-Calc, versão 4, utilizando-se o banco de dados TCFE5.

\section{RESULTADOS}

Para todos os sistemas de liga, a precipitação de compostos, a solubilização de elementos na austenita e a influência do $\mathrm{C}, \mathrm{N}, \mathrm{Nb}$ e $\mathrm{V}$ sobre a precipitação foram avaliadas a partir de diagramas de equilíbrio. Os diagramas da Figura I apresentam a quantidade de precipitados formados em função da temperatura, considerando o balanço de massa para I g de aço produzido, sendo FCC\#2, FCC\#3 e FCC\#4 referentes aos precipitados ricos em $\mathrm{Ti}$, $\mathrm{Nb}$ e $\mathrm{V}$, respectivamente. Nestes diagramas também estão mostrados os campos monofásico $(\gamma)$ e bifásico $(\gamma+\alpha)$. A fração mássica dos elementos $\mathrm{C}, \mathrm{N}, \mathrm{Ti}$, $\mathrm{Nb}$ e $\mathrm{V}$ em solução sólida na austenita em função da temperatura é mostrada na Figura 2. As temperaturas de início de precipitação dos compostos formados nos sistemas estão apresentadas na Tabela 2.
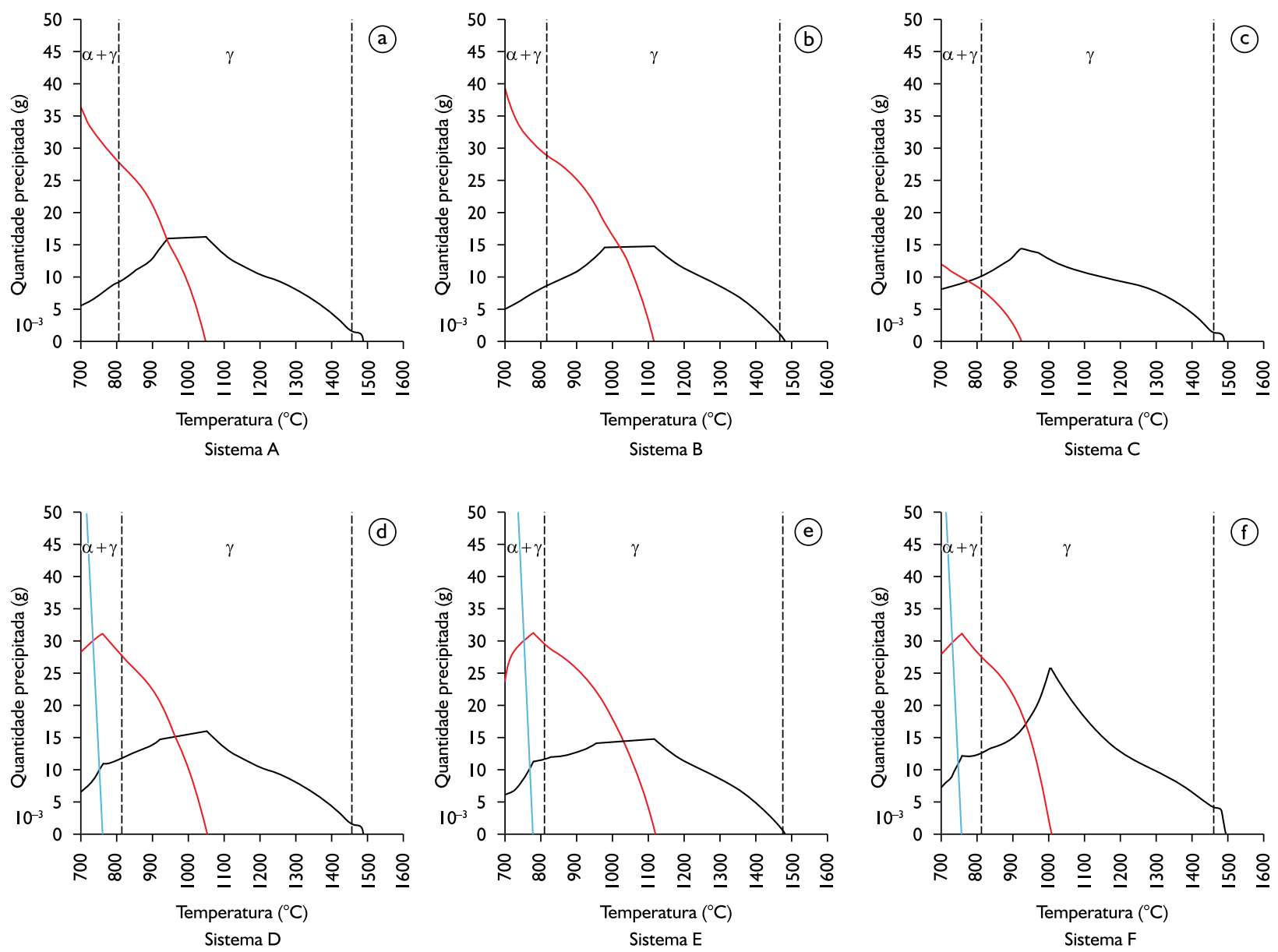

- FCC\#2, rico em $\mathrm{Ti}$ - FCC\#3, rico em Nb - FCC\#4, rico em $\mathrm{V}$

Figura I. Quantidade de precipitado formado em função da temperatura para os sistemas de liga estudados. $\gamma$ (austenita); $\alpha$ (ferrita). 
(a)

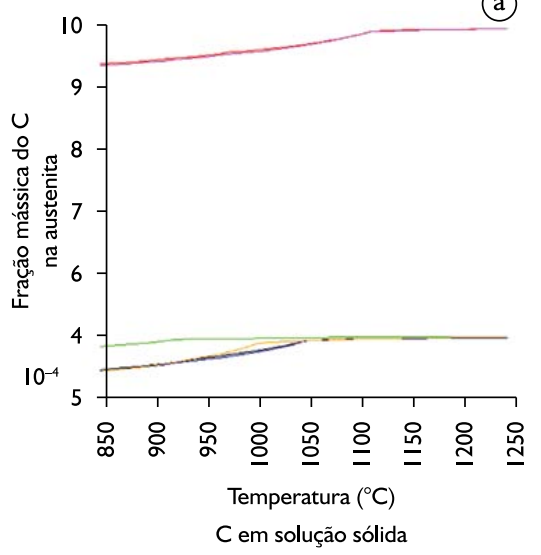

(b)

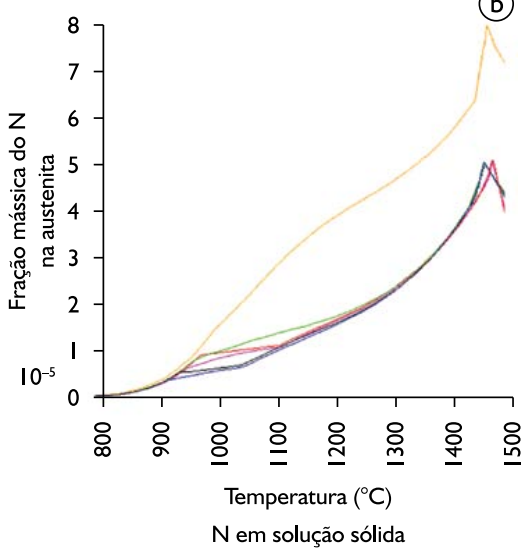

(d)

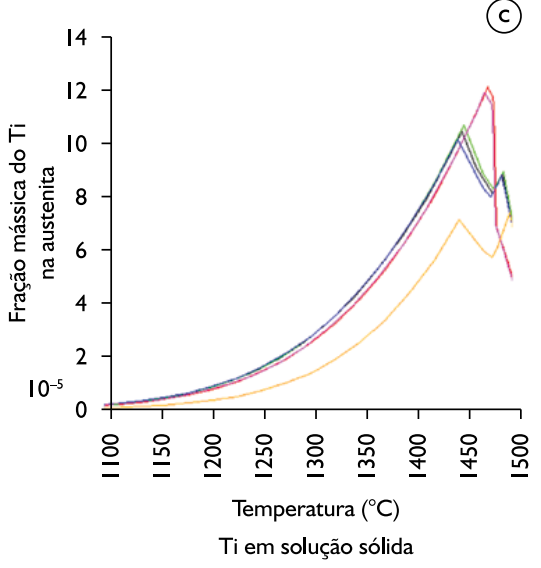

(e)
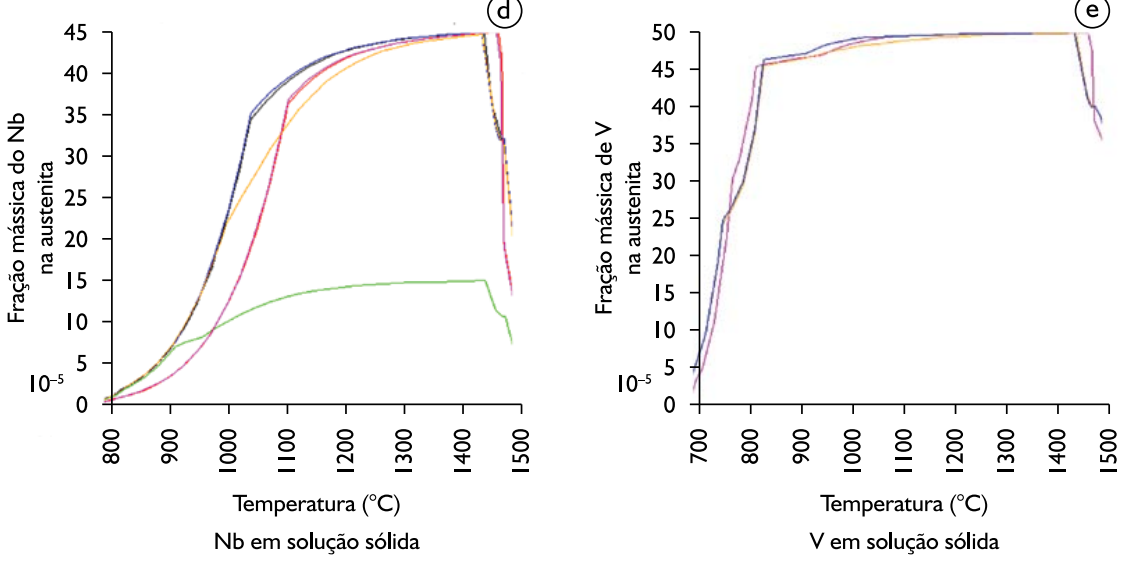

$-\mathrm{A}-\mathrm{B}-\mathrm{C}-\mathrm{D}-\mathrm{E}-\mathrm{F}$

Figura 2. Fração mássica do componente em solução sólida na austenita em função da temperatura.

Tabela 2. Temperatura de início da precipitação dos compostos rico em $\mathrm{Ti}$ (FCC\#2), rico em $\mathrm{Nb}$ (FCC\#3) e rico em $\vee$ (FCC\#4) para os sistemas A, B, C, D, E e F.

\begin{tabular}{lcccccc}
\hline Precipitado & \multicolumn{6}{c}{ Temperatura de precipitação, ${ }^{\circ} \mathbf{C}$} \\
\cline { 2 - 7 } & A & B & C & D & E & F \\
\cline { 2 - 7 } & 1485 & $148 \mid$ & 1486 & 1487 & 1479 & 1496 \\
\hline FCC\#2 & 1049 & 1115 & 922 & 1052 & 1117 & 1009 \\
FCC\#3 & - & - & - & 759 & 778 & 759 \\
FCC\#4 & - & \multicolumn{7}{c}{} \\
\hline
\end{tabular}

\section{DISCUSSÃO}

Os sistemas de ligas diferem entre si pelos teores de $\mathrm{C}, \mathrm{Nb}, \mathrm{N}$ e V. O sistema B apresenta o dobro do teor de $\mathrm{C}$ do sistema $\mathrm{A}$, sendo que o teor de $\mathrm{Nb}$ deles é três vezes maior que o do sistema $C$. Os sistemas $D$ e $E$ diferem dos sistemas $A$ e $B$, respectivamente, pela adição de $\mathrm{V}$. $\mathrm{O}$ sistema $\mathrm{F}$ apresenta a mesma composição química base do sistema $\mathrm{D}$, com exceção do teor de $\mathrm{N}$ que é I,6 vezes maior. De acordo com a Figura I, os precipitados ricos em $\mathrm{Ti}$ e $\mathrm{Nb}$ estão presentes em todos os sistemas estudados. Há possibilidade de formação do precipitado rico em $V$ nos sistemas D, E e F. Ressalta-se que todos estes precipitados são cúbicos de face centrada e apresentam solubilidade mútua entre si, podendo um precipitado conter simultaneamente $\mathrm{Ti}, \mathrm{Nb}, \mathrm{V}, \mathrm{C}$ e $\mathrm{N}$, sendo um ou dois elementos mais predominantes. Isto é considerado no modelo utilizado pelo Thermo-Calc, no qual os carbonetos e nitretos de $\mathrm{Ti}, \mathrm{Nb}$ e $\mathrm{V}$ apresentam a mesma estrutura cristalina, $(\mathrm{Ti}, \mathrm{Nb}, \mathrm{V})(\mathrm{C}, \mathrm{N}, \mathrm{Va})$, sendo Va referente às lacunas. ${ }^{(5)}$

$\mathrm{Na}$ Figura 2a, verifica-se a diminuição da quantidade de $\mathrm{C}$ dissolvido na austenita em temperaturas que correspondem ao início da formação dos precipitados ricos em $\mathrm{Nb}$, indicando que o precipitado FCC\#3 tende a corresponder ao $\mathrm{NbC}$. Analisando-se os diagramas referentes ao $\mathrm{N}$ e Ti em solução sólida na austenita (Figuras 2 b e $2 c$, respectivamente), observa-se que praticamente todo $\mathrm{Ti}$ se precipita em temperaturas superiores a I. $100^{\circ} \mathrm{C}$ e a quantidade de $\mathrm{N}$ dissolvido na austenita diminui consideravelmente a temperaturas acima de $1.200^{\circ} \mathrm{C}$. Essas evidências sugerem que em todos os sistemas de ligas estudados o precipitado $\mathrm{FCC \# 2}$ corresponde ao TiN. Devido à sua baixa solubilidade, a precipitação do nitreto de titânio normalmente inicia a temperaturas muito altas e é determinada pela composição química do 
aço, principalmente, pelos teores de $\mathrm{C}$ e N. ${ }^{(6,7)}$ Pelo diagrama da Figura 2c, pode-se inferir que a probabilidade de formação de TiN no aço líquido é maior no sistema $F$, pois apresenta menor fração mássica de Ti na austenita, indicando que parte deste elemento já se precipitou em temperaturas muito elevadas.

Conforme os dados das Tabelas I e 2, verifica-se que a adição de $\mathrm{C}$ abaixa a temperatura de precipitação do composto rico em $\mathrm{Ti}(\mathrm{TiN})$, o que é comprovado na Figura $2 c$, na qual está mostrado que nos sistemas $B$ e $E$ a solubilidade do Ti na austenita é maior. Por outro lado, o aumento do teor de $C$ diminui a quantidade de $\mathrm{Nb}$ dissolvido na austenita (Figura 2d), promovendo a precipitação do composto rico em $\mathrm{Nb}(\mathrm{NbC})$ a temperaturas mais elevadas. A precipitação do $\mathrm{TiN}$ a elevadas temperaturas é favorecida pelo aumento do teor de $\mathrm{N}$, que também abaixa a temperatura de precipitação do $\mathrm{NbC}$, pois promove a incorporação de átomos de $\mathrm{Nb}$ ao precipitado de $\mathrm{TiN}$, diminuindo a quantidade de $\mathrm{Nb}$ em solução sólida na austenita. As adições de $\mathrm{N}$ não têm efeito sobre a temperatura de precipitação do composto rico em $\mathrm{V}$, visto que não há mais $\mathrm{N}$ disponível em solução sólida na austenita. A diminuição do teor de $\mathrm{Nb}$ não exerce influência sobre a temperatura de precipitação do TiN, entretanto, diminuiu consideravelmente a do $\mathrm{NbC}$. $\mathrm{A}$ adição de $\mathrm{V}$ não altera as temperaturas de precipitação do $\mathrm{TiN}$ e nem do $\mathrm{NbC}$.

Para impedir o crescimento dos grãos austeníticos durante a etapa de reaquecimento, é necessária a presença de precipitados finos de $\mathrm{TiN}$, o que requer uma razão $\mathrm{Ti} / \mathrm{N}$ hipoestequiométrica na composição química do aço. Para uma quantidade fixa de $\mathrm{Ti}$, o aumento do teor de $\mathrm{N}$ diminui a solubilidade das partículas de $\mathrm{TiN}$, o que pode favorecer a formação destes precipitados no aço líquido. ${ }^{(7,8)}$ Esta observação foi verificada nos cálculos do Thermo-Calc para o sistema F, Figura 2.c. Entretanto, nos sistemas ligas estudados, a razão $\mathrm{Ti} / \mathrm{N}$ do sistema $\mathrm{F}$ é cerca de I,6 vezes menor do que a dos outros sistemas, e, segundo os cálculos termodinâmicos, a probabilidade de formar precipitados grosseiros de TiN é maior neste sistema. Essa contradição pode ser explicada pela cinética, que atua na nucleação, distribuição e no tamanho dos precipitados. ${ }^{(9)}$

A partir das análises das curvas de $\mathrm{C}, \mathrm{NeN}$ em solução sólida na austenita, pode-se inferir que uma parcela do $\mathrm{Nb}$ adicionado se precipitará junto ao TiN, visto que há indícios de precipitação de $\mathrm{Nb}$ a altas temperaturas. $\mathrm{O} \mathrm{Nb}$ restante se combinará preferencialmente com o $\mathrm{C}$ para formar $\mathrm{NbC}$, pois praticamente não há mais $\mathrm{N}$ disponível em solução sólida, exceto no sistema $\mathrm{F}$. É desejável que grande parte do $\mathrm{Nb}$ esteja dissolvido na austenita durante o encharque e, portanto, disponível para o refino de grão e endurecimento por precipitação nas etapas posteriores do processamento a quente do aço. Pelos cálculos do Thermo-Calc (Figura 2d), para os sistemas com o mesmo teor de $\mathrm{Nb}$, observa-se que nas temperaturas entre $1.100^{\circ} \mathrm{C}$ e $1.200^{\circ} \mathrm{C}$ - faixas de temperatura de encharque - a quantidade de $\mathrm{Nb}$ não solubilizada será maior nos sistemas B, E e F.

Com relação ao $\mathrm{V}$ em solução sólida na austenita (Figura $2 \mathrm{e}$ ), observa-se que o início da precipitação deste elemento ocorre a temperaturas inferiores a $1300^{\circ} \mathrm{C}$, indicando o enriquecimento do precipitado rico em $\mathrm{Nb}$ com uma parcela do V. Pela Figura 2b, verifica-se que a quantidade de $\mathrm{N}$ disponível em solução sólida na austenita é muito pequena em temperaturas a partir das quais ocorre a precipitação acentuada do $\mathrm{V}$ (aproximadamente $830^{\circ} \mathrm{C}$ ).
Portanto, a formação de VN é pouco provável, ficando assim o $\mathrm{V}$ em solução sólida. ${ }^{(10)}$ Pode-se inferir a possibilidade de precipitação do VC, pois os cálculos do Thermo-Calc mostram que a temperatura de início de precipitação do composto rico em $\mathrm{V}$ (FCC\#4) ocorre a temperaturas inferiores a $800^{\circ} \mathrm{C}$ (Tabela 2).

Para maximizar o efeito das microadições, é necessário ter algum conhecimento sobre o fenômeno da precipitação durante o processamento do aço. As relações de produto de solubilidade disponíveis na literatura descrevem de forma simplificada as curvas solvus de sistemas ternários, além de não considerarem as interações entre os solutos. ${ }^{(11,12)}$ A termodinâmica computacional permite prever, com maior exatidão do que as equações de produto de solubilidade, as condições de precipitação de elementos microligantes em sistemas multicomponentes, pois as interações entre os solutos são consideradas. Portanto, é um importante método de otimização para a definição de projetos de liga e das condições de processamento termomecânico. Todavia, ressalta-se que, na prática, as informações obtidas com os cálculos termodinâmicos dependem de uma cinética favorável para serem procedentes.

\section{CONCLUSÕES}

O estudo da precipitação de microligantes em sistemas de liga de aços ARBL, feita no Thermo-Calc, forneceu informações qualitativas importantes sobre este fenômeno. De acordo com os cálculos termodinâmicos, existe a possibilidade de formação de precipitados ricos em $\mathrm{Ti}$, $\mathrm{Nb}$ e/ou $\mathrm{V}$ nas ligas estudadas, sendo a precipitação determinada pela composição química do aço, principalmente, pelos teores de $\mathrm{C}$ e $\mathrm{N}$.

A termodinâmica computacional se constitui, pois, em importante ferramenta no desenvolvimento de projetos de liga, permitindo o estudo do comportamento termodinâmico de sistemas multicomponentes, o que auxilia na definição de parâmetros das etapas de processamento termomecânico. Entretanto, a análise termodinâmica não deve ser considerada isoladamente, visto que a formação de fases e/ou compostos depende de uma cinética favorável.

\section{Agradecimentos}

Os autores agradecem ao Prof. Dr. André Luiz Vasconcellos da Costa e Silva, da EEIMVRUFF pelo apoio dado durante a elaboração do presente trabalho. 


\section{REFERÊNCIAS}

I LIU, Z. K. Thermodynamic calculation of carbonitrides in microalloyed steels. Scripta Materialia, v. 50, n. 5, p. 60I-6, Mar. 2004.

2 KEOWN, S. R.; WILSON, W. G. Prediction of precipitate phases in microalloy steels containing niobium, carbon, nitrogen and aluminum. In: THERMOMECHANICAL PROCESSING OF MICROALLOYED AUSTENITE, I982, p. 343-59.

3 NAGAl, Y. et al. YS $500 \mathrm{Nmm}^{2}$ High strength steel for offshore structures with good CTOD properties at welded joints. Nippon Steel Technical Report, n. 90, p. 14-9, July 2004.

4 ANDERSSON, J. O. et al. Thermo-Calc \& Dictra: computational tools for materials science. Calphad, v. 26, n. 2, p. 273-3/2, jun. 2002.

5 PINDOR, L. et al. Investigation into secondary phases in steels microalloyed with vanadium and nitrogen. Ironmaking and Steelmaking, v. 35, n. 2, p. 124-8, Feb. 2008.

6 ADRIAN, $\mathrm{H}$. Thermodynamic calculation of carbonitride precipitation as a guide for alloy design of microalloyed steels. In: MICROALLOYING '95 CONFERENCE, Pittsburgh. Proceedings... [S.I.]: Iron and Steel Society, 1995. p. 285-305.

7 HERMAN, J. C.; MESSIEN, P.; GRÉDAY, T. HSLA Ti - containing steels. In: INERNATIONAL CONFERENCE ON THERMOMECHANICAL PROCESSING OF MICROALLOYED AUSTENITE, 1982, Pittsburgh. Proceedings... Warrendale, PA: Metallurgical Society if AIME, [s.d.]. p. 655-7I.

8 VERVYNCKT, S. et al. Austenite recrystallization-precipitation interaction in niobium microalloyed steels. ISIJ International, v. 49, n. 6, p. 91 I-20, Feb. 2009.

$9 \mathrm{CHOI}$, J. K. Development of high strength and high performance steels at Posco Through HIPERS-2I Project. POSCO, Pohang, Korea.

10 XIONG, Y. et al. Study on high tensile heavy plate with V-N microalloying technology. In: INTERNATIONAL SEMINAR 2005 ON APPLICATION TECHNOLOGIES OF VANADIUM IN FLAT - ROLLED STEELS, Beiijing. Suzhou. China: A Vanitec, 2005. p. 64-8.

II SILVA, A. L. V. C.; AVILLEZ, R. R.; CORDEIRO, N. B. Modelos para a solubilidade de precipitados relevantes para a laminação a quente de aços. In: CONGRESSO ANUAL DA ABM, 54., 1999, São Paulo. Anais... São Paulo: ABM, 1995. p. 457-67.

12 ZAJAC, S.; LAGNEBORG, R.; SIWECKI, T. The role of nitrogen in microalloyed steels. In: MICROALLOYING '95 CONFERENCE, Pittsburgh. Proceedings... [S.I.]: Iron and Steel Society, 1995. p. 32I-38.

Recebido em: 27/10/2010

Aprovado em: $13 / 12 / 2010$ 